\title{
Delivering on the promise of universal health coverage
}

\author{
A new initiative to focus on improving healthcare delivery systems
}

\author{
Ashish Jha director ${ }^{1}$, Fiona Godlee editor in chief $^{2}$, Kamran Abbasi international editor ${ }^{2}$ \\ ${ }^{1}$ Harvard Global Health Institute, Cambridge, MA 02138, USA ; ${ }^{2}$ The BMJ, London, UK
}

Universal health coverage - the notion that people should be able to access healthcare services regardless of their ability to pay, and do so with financial protection-is a major focus for G7 policy makers and others around the globe. It is an important component of the sustainable development goals, leading many countries to increase their use of scarce public resources to ensure their citizens are covered. The motivation, of course, is compelling. People should not develop or die from preventable or treatable conditions because they are poor, and treatment should not lead to financial bankruptcy.

Simply prioritising universal health coverage, however, will not be enough to achieve its main goals. Even if nations are able to identify the necessary resources, fundamental questions about achieving effective universal coverage remain unanswered. Without these answers, simply pushing for universal coverage may waste precious resources without achieving the important goals of improved health and wellbeing of citizens in a way that is efficient for people and countries.

Although the primary focus is coverage (financing healthcare services, ensuring adequate staff, etc), success depends on the underlying healthcare delivery system. It also requires clarity about the trade-offs. Governments have limited resources but many important priorities. What will not be funded because governments focus on universal coverage? Even in Massachusetts, one of the wealthier US states, investments in achieving universal health coverage for its population of seven million people coincided with substantial reductions in spending on public health, education, and infrastructure. ${ }^{1}$

If policy makers decide that universal health coverage is worth supporting, they will need better information on how to implement it. How should investments be structured? Should it all be public funding or should governments fund private insurance and private providers? How should governments ensure that there is a legal and regulatory framework to manage the legal rights that such programmes might confer? And, of course, how is it possible to ensure that the coverage that is created is worth having? As Teerawattananon and colleagues have pointed out, setting priorities early helps nations make better decisions about how best to achieve these goals, especially when faced with scarce resources. ${ }^{2}$
Herein lies one of the largest challenges of universal health coverage. The safety, quality, and efficiency of most healthcare delivery systems (across high, middle, and low income countries) are far from the best they could be. In many countries, especially those with scarce resources, the quality of the underlying healthcare delivery system is so poor that it is unclear whether increasing access to services will do more good or more harm. Conservative estimates put unsafe medical care as one of the top 10 causes of human harm, with adverse medical events affecting $8-15 \%$ of hospital inpatients in high income countries and even more in low and middle income countries. ${ }^{3}$ In some places, patients who visit physicians are more likely to get the wrong diagnosis and harmful treatment than they are to get the right ones. $^{45}$

While we strongly support the idea of universal health coverage, we do not know how to ensure we get the most for our investments. We do know, however, that given the millions of deaths that occur from poor quality care, one way to improve the value of that investment is to improve the quality of the underlying delivery system.

To this end, the Harvard Global Health Institute and The BMJ are partnering to launch a new initiative on effective universal coverage. We agree with the underlying goals - that everyone around the world deserves access to healthcare services when they are ill and encounters with the healthcare system should make people better without bankrupting them. But there is a vast gap between those two goals and our ability to deliver them. That vast gap is mainly due to deficiencies in knowledge about both optimal approaches to financing and effective models for healthcare delivery. So little is known about how to do this well-partly because each nation is unique with a different set of needs and a different path to achieving true, effective universal health coverage. We also know little about how best to deliver what is known to work. But that doesn't mean that there aren't generalisable principles, and our hope is that The $B M J$ can be a vehicle for furthering our knowledge about how to do universal health coverage well.

We welcome articles for consideration that help us further our knowledge and insights. We are willing to consider original 
research papers as well as analyses and opinion pieces. Themes of interest include

- Efforts to improve effectiveness of health services in low and middle income countries

- Comparative analyses of financing schemes

- The intended and unintended effects of policy level interventions

- Scale-up and broader applicability of delivery strategies

- Legal mechanisms that aim to improve accountability

- Measurement strategies for complex delivery environments. We are at a critical juncture in global health. The world has increasingly come to realise that we are interdependent and that a poor performing health system in one place is a threat to us all. We must pull together to help nations develop their own healthcare systems and achieve effective universal coverage in ways that are consonant with their history, culture, and values.
We can all learn from each other, and learn we must, because good intentions are a start but they are insufficient.

Competing interests: We have read and understood BMJ policy on declaration of interests and have no relevant interests to declare.

Provenance and peer review: Commissioned; not externally peer reviewed.

1 Massachusetts Budget and Policy Center. Massachusetts state budget. 2016. http:// massbudget.org/browser/index.php.

2 Teerawattananon Y, Luz A, Kanchanachitra C, Tantivess S. Prince Mahidol Award Conference secretariat. Role of priority setting in implementing universal health coverage. BMJ 2016;532:i244. doi:10.1136/bmj.i244 pmid:26813049.

3 Jha AK, Larizgoitia I, Audera-Lopez C, Prasopa-Plaizier N, Waters H, Bates DW. The global burden of unsafe medical care: analytic modelling of observational studies. BMJ Qual Saf 2013;22:809-15. doi:10.1136/bmjqs-2012-001748 pmid:24048616.

4 Das J, Hammer J, Leonard K. The quality of medical advice in low-income countries. $J$ Econ Perspect 2008;22:93-114.

5 Das J, Holla A, Das V, Mohanan M, Tabak D, Chan B. In urban and rural India, a standardized patient study showed low levels of provider training and huge quality gaps. Health Aff (Millwood) 2012;31:2774-84. doi:10.1377/hlthaff.2011.1356 pmid:23213162.

Published by the BMJ Publishing Group Limited. For permission to use (where not already granted under a licence) please go to http://group.bmj.com/group/rights-licensing/ permissions 